\title{
Evaluación de los aprendizajes en escenarios clínicos: ¿Qué evaluar y por qué?
}

\author{
Learning assessment in clinical settings. What assess ? Why?
}

\author{
Orlando Maroto-Marín ${ }^{1}$ \\ Universidad de Costa Rica \\ Facultad de Odontología \\ Facultad de Educación \\ San José, Costa Rica \\ orlandomaroto@gmail.com \\ ORCID: orcid.org/0000-0002-9057-0976
}

\begin{abstract}
Recibido: 15 mayo 2015 Aceptado: 22 setiembre 2016 Corregido: 15 octubre 2016
\end{abstract}
\begin{abstract}
Resumen: Este material corresponde a un ensayo en el que se analiza el proceso de enseñanza en el escenario clínico como espacio de aprendizaje, específicamente, en las carreras del área de la salud. Además, se incluyen aspectos claves de la enseñanza en la clínica $y$, por ende, la correspondiente evaluación de los aprendizajes. Se pretende propiciar la actividad reflexiva en el campo docente del área de salud, para una mejor comprensión de esta por parte del profesorado y relacionar la evaluación como un aspecto importante en el aprendizaje clínico. Se va más allá de una concepción tradicional de la evaluación, asumida como medición, cuyo objetivo es brindar solamente la calificación para determinar el nivel de rendimiento y la promoción del estudiantado. Se exponen algunos aspectos a evaluar dentro del proceso formativo y la importancia de una adecuada retroalimentación para el grupo de estudiantes que participan en estos cursos en particular.
\end{abstract}

Palabras claves: Aprendizaje clínico, evaluación educativa, evaluación clínica, docencia en salud.

\begin{abstract}
This analysis corresponds to a trial in which the teaching process is analyzed in the clinical setting as a learning space. In specific, in the majors related to health. In addition, key aspects of teaching in the clinic and thus the corresponding assessment of learning are included. It aims to encourage reflective activity in the educational field in the health area to enhance a better understanding of this process by teacher. Also, it will help to link evaluation as an important aspect of clinical learning beyond a traditional conception of evaluation, assumed as measurement, which aims to provide a rating to determine the level of performance and promotion of students. Some aspects to be evaluated within the teaching process and the importance of adequate feedback to the group of students who enroll these courses in particular are exposed.
\end{abstract}

Keywords: Clinical learning, educational assessment, clinical assessment, health teaching.

1 Orlando Maroto Marín, Licenciado en Odontología, Universidad de Costa Rica, Licenciado en Docencia, Universidad Estatal a Distancia, Máster en Tecnología Educativa, Universidad Estatal a Distancia, Máster en Currículo y Docencia Universitaria, ULACIT. Experiencia docente por 20 años, laboró en la Facultad de Odontología, UCR, y la Escuela de Formación Docente, Departamento de Docencia Universitaria, UCR. Algunas obras: El uso de presentaciones digitales en la educación superior: una reflexión sobre la práctica. Actualidades investigativas en Educación. Docencia de Clínicas de Odontología: un acercamiento hacia el perfil de sus docentes. ODOVTOS. El portafolio digital utilizado en la evaluación de la clínica de odontología en la Universidad de Costa Rica (UCR): Reporte de una experiencia. Actualidades Investigativas en Educación. Guías de tratamiento para fomentar el aprendizaje significativo en la Clínica Dental de Docencia. ODOVTOS. Currículo por competencias: Una alternativa para la enseñanza de la Odontología. ODOVTOS. 


\section{Introducción}

Las estudiantes y los estudiantes que se forman en las carreras de Ciencias de la Salud, en la Universidad de Costa Rica, como lo son: Medicina, Odontología, Enfermería, Microbiología, Nutrición, Farmacia, y las llamadas Tecnologías en Salud, de la Universidad, tienen durante su formación una serie de cursos que involucran experiencias de aprendizaje clínicas con actividades supervisadas que abarcan la atención de pacientes. Por medio de estas, el estudiantado refuerza aquellos procederes que les permiten tratar a las personas que acudan a los servicios de salud de la mejor manera posible, conjugando no solo el bagaje cognitivo y procedimental conseguido en niveles anteriores, con los nuevos conceptos por aprender, sino también los procedimientos hasta ahora aprendidos a nivel teórico y práctico, al iniciar su utilización con pacientes. El estudiantado de estos cursos, en estos espacios desarrollan las habilidades y destrezas que les permitirán ejercer su profesión y continuar realizándose y creciendo en su campo disciplinario.

Este ensayo tiene como objetivo exponer un análisis de las características de los escenarios clínicos de enseñanza: por qué evaluar y qué evaluar en ellos. Se entiende, por escenarios, aquellos que involucran una práctica supervisada en atención de pacientes con la guía del personal docente, quien tiene un rol dual como profesora o profesor y clínica o clínico. Usualmente este personal docentes no posee formación en el campo de la pedagogía, por lo cual, con el análisis propuesto en este ensayo, se pretende despertar su interés en el tema evaluativo como parte del proceso de enseñanza, en este nivel de docencia clínica, y cooperar en el análisis reflexivo de la pedagogía en ciencias de la salud.

\section{Escenarios clínicos de aprendizaje}

En este apartado se abarcará la descripción de un escenario clínico de aprendizaje. Para ello se empieza por definir qué es clínica. La palabra clínica viene del griego Kliniké, lo que se relaciona con la atención médica de pacientes en la cama (Diccionario Etimológico, 2015). Además, es definido como el "ejercicio práctico de la medicina relacionado con la observación directa del paciente y con su tratamiento" (Diccionario de la Real Academia Española, 2015, p. 5). Como se puede leer en las citas anteriores, el término se refiere a la atención de pacientes e involucra el ámbito médico. Para efectos de este ensayo se relacionará con la atención de pacientes por profesionales de la salud en sus diferentes campos.

Entonces, ¿qué es un escenario clínico de aprendizaje? Este espacio es considerado por el autor como el "aula clínica" donde cada discente se enfrenta a experiencias de aprendizaje que involucran el ejercicio supervisado de la profesión que se estudia y no las prácticas de simulación. En este caso, se relaciona con los hospitales, clínicas de servicio, clínicas para la docencia, entre otros. En estas, dependiendo de la carrera que se trate, no se atienden, exclusivamente, las necesidades de pacientes que padecen una enfermedad, sino que, además, a quienes se han involucrado en los programas preventivos en salud.

Estos espacios de aprendizaje clínicos son considerados esenciales en la preparación de profesionales de la salud, ya que permiten lograr habilidades, destrezas y actitudes que 
difícilmente se aprenderían de otra forma (Lidjedal, Engqvist, Porthén y Bolander, 2014). Este tipo de enseñanza está relacionada con el aprendizaje en el trabajo (Durante, 2012), es decir, en ambientes de trabajo de la profesión. Cada paciente que llega a la clínica, según Álvarez, Gulgelmeier y Hermida (2013), “... representa una situación de salud única, en condiciones reales..." (p. 5), es decir, con las condiciones que involucran el ambiente de trabajo para el cual el estudiantado se está preparando.

Estudiantes de las carreras de la salud suelen pasar por diferentes niveles durante su formación profesional, tal como se esquematiza por el autor de este ensayo en la figura 1.

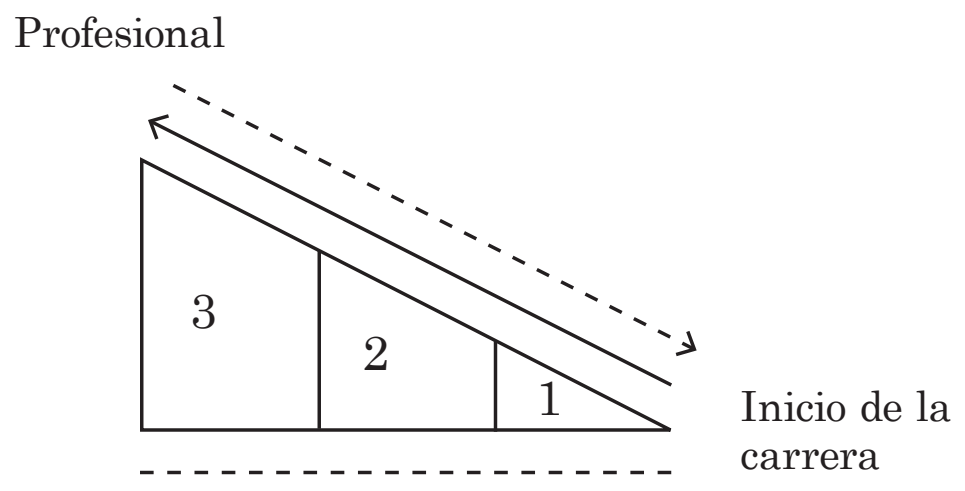

Figura 1. Niveles de preparación generales en las carreras de la salud. Elaboración propia.

En la figura 1, el inicio de la carrera, la primera parte del triángulo (1), representa el tiempo durante el cual el estudiantado aprende los nuevos términos e ideas, la mayoría del tipo de contenido declarativo, esto es, los conocimientos, los hechos, los conceptos y los principios (DíazBarriga y Hernández, 2007), y aunque las materias suelen ser semejantes entre las diferentes áreas de la salud, los niveles de profundización varían en cada una y de acuerdo con el objeto de estudio de cada disciplina. En la mayoría de estas predominan aún las recomendaciones de Flexner, quien afirmaba que la atención médica debía basarse en el conocimiento de las ciencias biomédicas, entre otras: anatomía, fisiología y farmacología (Borrell, 2005).

En la segunda parte (2) de la figura 1, se representan aquellas prácticas de laboratorio en ambientes ideales o aquellas experiencias en las que se utilizan simuladores que proveen, al estudiantado, la oportunidad de practicar lo necesario para aprender la habilidad requerida, o desarrollar las destrezas, repitiendo los procedimientos tantas veces como sea necesario antes de ponerlas en práctica. Así se permite el mejoramiento continuo y se aprende de los propios errores, como afirma Escalante y Matos (2013). Con el uso de los simuladores, además, el personal docente puede aislar el proceso de enseñanza de la realidad en el cuidado de una persona, evitándose en esta práctica la tensión e impredictibilidad de la clínica. Es en esta fase donde se aprenden las bases de los conocimientos procedimentales, es decir, de los procedimientos que son las llamadas habilidades y destrezas (Díaz- Barriga y Hernández, 2007). En todas las profesiones, los laboratorios son variados y, si utilizan las simulaciones, 
estas abarcan diferentes niveles que pueden pretender diversos grados de experticia. (Por ejemplo, los simuladores de alta fidelidad).

En la figura 1, en la tercera parte del triángulo (3), están representadas las experiencias de aprendizaje clínicas cuyo desarrollo, según el autor de este ensayo, se da en los escenarios clínicos, los cuales se encuentran generalmente cercanos al final de la carrera e involucran aquellas experiencias en la práctica, donde los otros conocimientos se aplican en la acción profesional supervisada. En este nivel se requiere, además, que el estudiantado complemente el desarrollo del componente actitudinal, el cual es especialmente importante al referirse a la comunicación con el sujeto paciente y la ética en el servicio profesional. En esta fase el estudiantado desarrolla la habilidad del conocimiento clínico, en el cual, según Boshuiizen, Norman y Schmidt (1990), se encapsula el conocimiento biomédico, lo que sucede al desarrollarse la experticia. Aunque este ensayo se refiere de preferencia al nivel de grado profesional, los posgrados podrían ser una extensión de este último nivel (3).

En la figura 1 se ha colocado también una línea continua que muestra la direccionalidad del proceso formativo y, asimismo, las líneas discontinuas que proponen la integralidad necesaria entre los cursos y la no fragmentación del conocimiento, esta última asociada más al paradigma asociacionista (Durante, 2012). El aprendizaje en la práctica clínica se caracteriza por el desempeño de tareas y la resolución de problemas en un ambiente donde se reflejan las diferentes formas en que utilizará el estudiantado sus conocimientos, en la futura práctica profesional (Stailmeijer, Dolmans, Wolfhagen, y Scherpbier, 2009).

La enseñanza en estos espacios constituye una estrategia esencial en las carreras de la salud (Durante, 2012; Liljedahl, Engqvist, Porthén, y Bolander, 2014), en las que es necesario no solo preocuparse por la adquisición de conocimientos por la parte estudiantil, sino también por adquirir las habilidades y alcanzar el conocimiento de su aplicación (Taylor, Grey, y Satterthwaite, 2013).

Como afirman Lucarelli et al. (2009), esta práctica posee ciertas características distintivas en relación con otras, como son la variabilidad, la impredictibilidad y, además, la inmediatez en la toma de decisiones. Los retos en la enseñanza en la clínica se relacionan con la presión del tiempo, la diferencia entre el alumnado y las necesidades de sus pacientes, todo dentro de un contexto específico.

Algunas veces se atribuye a la docencia clínica el carácter de oportunista, donde el planeamiento es más difícil, pues no siempre se presentarán casos modelos de los diferentes tratamientos o la posibilidad de realizar exámenes y no siempre existe suficiente cantidad de pacientes para que todo el alumnado les atienda y cumpla con los requisitos en un tiempo lectivo establecido. Según los aspectos mencionados, el planeamiento de la experiencia clínica es difícil, lo que podría complicarse más, si el curso es impartido por variedad de docentes. El contexto del cuidado de la salud es reconocido como incierto por Higgs y Titchen (2001) y en continuo cambio, según Ryan et al. (2003) (ambas referencias citadas por Delany y Molloy, 2009).

En el proceso de enseñanza- aprendizaje ocurrido en escenarios clínicos participan tres personas, a saber: discente, docente y la persona atendida (Lucarelli et al. 2009). Ello corresponde a lo que se llama la tríada del aprendizaje clínico (Álvarez, Rojas, Navas y Quero, 2011). Estos detalles potencian la complejidad del proceso de enseñanza- aprendizaje 
clínico. No solo se deben considerar las necesidades de aprendizaje del estudiantado, sino también las necesidades que presenta la persona atendida, quien a veces tiene expectativas diferentes a las posibles de alcanzar en la atención en una clínica docente, aun cuando todo tratamiento se brinde con la supervisión del equipo docente, quien desempeña dos papeles simultáneamente: de docente y de clínico, pues es el personal responsable; además, en algunos espacios educativos clínicos, con un servicio asistencial de recargo o viceversa con el recargo de docencia. Incluso, son estas mismas personas quienes diseñan la evaluación y la administran en estas prácticas. Esto podría inducir a la subjetividad y afectar la credibilidad y fiabilidad del proceso de evaluación y causar cierta frustración de parte del estudiantado al calificar los procesos de arbitrarios (Taylor, Grey y Satterthwaite, 2013).

Otro aspecto a considerar es que la docencia universitaria generalmente es impartida por profesionales de áreas no propias de la carrera de Educación, y la gente en este caso es entrenada por años en el área de su ocupación, pero no en la docencia.

Después de caracterizar los escenarios de aprendizaje en la clínica, el autor de este ensayo propone que, para la enseñanza clínica, son necesarios varios elementos, además del conocimiento específico del área profesional de parte del cuerpo docente. Uno de ellos es el reconocimiento de las características del proceso formativo desde el punto de vista didáctico para que se pueda rescatar, a pesar de la variabilidad y la falta de predictibilidad para el planeamiento educativo en esta práctica, el que se den los elementos necesarios que propicien el aprendizaje, lo cual requiere la instrucción, además de la evaluación con una retroalimentación conducente al desarrollo de las habilidades del estudiantado en un marco de atención seguro para las pacientes y los pacientes.

En el espacio de enseñanza clínico, se requiere también el modelaje de roles, el cual debe ser intencionalmente didáctico y esto lo afirma el autor de este artículo basado en la teoría de Bandura, quien señala la importancia de observar en la experiencia práctica a otros sujetos, especialmente aquellas que poseen cierto grado de significatividad dentro del proceso de aprendizaje. Inclusive el modelaje de roles permitirá relacionar el andamiaje cognitivo que ya posee el alumnado con las nuevas experiencias que ofrece la clínica, y permitir, así, al estudiantado un proceso de socialización dentro de su profesión, además de la identificación de su rol como aprendiente (Liljedal, Engqvist, Porthén y Bolander, 2014).

\section{¿Por qué evaluar?}

Inicialmente para tratar de contestar esta interrogante es necesario lograr un acercamiento hacia el significado del término evaluación, el cual, dentro de una concepción más tradicional, se suele relacionar con la medición, dejando de lado su papel formador dentro del proceso enseñanza aprendizaje, al asociarlo, a veces, a exámenes y al circunscribir su importancia únicamente a los resultados obtenidos por el alumnado (Moreno, 2009). Sin embargo, "evaluar es atribuir un valor al resultado y a la actuación de los alumnos en el contexto de una estimación académica de sus rendimientos" (Bruera, 2008, p. 603). El valor proviene de la estimación reflejada en una calificación, por tanto, la evaluación se va a limitar a una medición (Bruera, 2008). Se presenta la relación entre aquellas expectativas de 
aprendizaje que se establecen en un curso y los logros manifestados por el alumnado a partir de la realización de las actividades de evaluación propuestas por el profesorado.

Es importante acotar que la evaluación, dentro del proceso de aprendizaje, relaciona ambos términos, la evaluación y el aprendizaje, en una estrecha correlación; debido a ello, no es conveniente hablar de estos por separado, ya que el objeto de estudio de este tipo de evaluación es el aprendizaje (López y Hinojosa, 2001). La evaluación no es un apéndice de la enseñanza o del aprendizaje: es parte de ellos (Camillioni, Celman y Litwin, 1998). La evaluación tiene sus usos dentro del proceso de enseñanza, sin embargo, para promover el aprendizaje durante todo el curso, esta debe ser formativa; además sumativa, ya que es importante demostrar, si se alcanzó la competencia necesaria para promoverse en un curso y así laborar en la clínica atendiendo pacientes. Este es un aspecto sustantivo en la docencia universitaria y es necesario tomarlo en cuenta para todas las carreras y, por supuesto, para las del área de la salud, objeto de este ensayo.

La efectividad de la evaluación depende de su uso, lo que puede variar en importancia (Sweet, Hutly y Taylor, 2003), por ejemplo: para promover al alumnado, para buscar la calidad en la formación de profesionales y para realizar las variaciones necesarias desde la docencia. La evaluación es necesaria, entre otras cosas, para certificar al sujeto profesional que se está graduando, para dar retroacción, evaluar los programas formativos, para confirmar los valores, estándares y por la responsabilidad que se tiene ante la sociedad (Brailovsky, 2001).

La evaluación de los aprendizajes no corresponde solamente a la valoración final del proceso de enseñanza- aprendizaje. No debe pretenderse solamente aprobar o reprobar a una estudiante o a un estudiante, la retroalimentación pertinente puede propiciar, además, la mejora continua. Si bien es cierto que la función asociada con la certificación es muy valiosa dentro del contexto educativo, como ya se mencionó anteriormente, la evaluación de los aprendizajes conlleva otras funciones importantes a considerar. Una de ellas, como se ha venido citando, es la formativa, es decir, aquella que ofrezca información para mejorar el desempeño estudiantil.

Por su parte, Flores, Contreras y Martínez (2012) hacen alusión a la evaluación formativa como:

... parte del proceso de enseñanza en sus diferentes etapas, de acuerdo con Stufflebeam, es una evaluación permanente que va monitoreando los objetivos del curso y resultados de aprendizaje esperados, proporciona información que brinda elementos para valorar los avances del grupo; permite la realización de ajustes para la mejora de los cursos y para realimentar al estudiante sobre sus progresos. (p. 44)

Además, la evaluación formativa, como menciona López y Hinojosa (2001): “... se obtiene durante el proceso de enseñanza aprendizaje, para mejorarlo y dirigirlo...” (p. 27). Este tipo de evaluación se realiza durante todo el proceso de enseñanza aprendizaje y puede permitir al estudiantado desarrollar la metacognición; esta se entiende como la autopercepción y la percepción compartida de lo que el estudiantado sabe o de aquello que reconoce sobre lo aprendido (Delgado, 2009). Esta autopercepción se puede lograr mediante la auto evaluación, 
que puede ser propiciada por aquellas herramientas de evaluación que permitan la reflexión, y esta puede facilitar la comprensión de situaciones complejas y alcanzar el aprendizaje a partir de las prácticas (Man, Gordon y Macleod, 2009). La reflexión no se da en todo momento, se estimula más por los casos clínicos complejos (Man, Gordon y Macleod, 2009).

El reflexionar sobre la propia práctica impulsa la metacognición, que está cambiando el paradigma de aprendizaje en la clínica, esto, desde el tipo de estudiante que aplica lo que aprendió en el salón de clases al tipo de estudiante que practica efectivamente la medicina y aprende de sus experiencias (Quirk, 2006). Otro elemento a desarrollar, además de la metacognición, es la intuición, para así alcanzar la experticia en la clínica. Para conseguir esta experticia se requieren también algunas características propias del estudiantado, como la autoconfianza y la tolerancia a lo desconocido, entre otros (Quirk, 2006). En la clínica, la práctica debe considerarse como la parte principal de la educación y como aquello que ayudará a conseguir la experticia. Además, el abordaje de estas experiencias determinará el nivel con que se alcancen la metacognición y la intuición (Quirk, 2006). El estudiantado debe estar preparado para aprender de las experiencias y evaluar los resultados (Quirk, 2006). El desarrollo de las capacidades metacognitivas será un paso fundamental para la constitución del razonamiento clínico, que en su momento permitirá al estudiantado tomar decisiones y resolver problemas en su campo de estudio.

Entonces, que a partir de lo discutido hasta ahora, se puede responder qué es necesario evaluar en la clínica, no solo para certificar la aprobación de un curso, sino además para promover la metacognición y también para proveer de la información oportuna que pueda permitir la mejora constante del desempeño estudiantil. Esta información igualmente puede ser útil para el personal docente, al analizar la pertinencia de las acciones educativas realizadas en el curso.

Es necesario, también, señalar que no solo se debe conocer el para qué se evalúa sino que se debe considerar, con cuidado, el diseño de los instrumentos para recolectar información en la evaluación de una competencia clínica, por ejemplo, criterios como "la confiabilidad, la validez, el impacto educativo, la aceptabilidad por los distintos sujetos usuarios, y los costos eventuales" (Brailovsky, 2001, p.6) son importantes. Además de lo anterior, Santamaría, (2005), agrega para las pruebas escritas, orales o de ejecución, en general, se deben considerar, entra otros elementos, la objetividad y la representatividad. El primero referido a que la prueba debe funcionar independiente al juicio de la persona que evalúa y el segundo a que los contenidos vistos en clases deben estar representados con cierto equilibrio en la prueba. A continuación, se tratarán los aspectos de confiabilidad y de validez de las pruebas.

La confiabilidad se relaciona con que las pruebas estén diseñadas de tal manera que, al aplicarlas en situaciones similares, se obtengan resultados semejantes; en el caso de la evaluación clínica, esto requiere especial atención. Tal como afirma Brailovsky (2001), se puede dar lo que él reconoce como el fenómeno de la especificidad de la situación clínica, que “...implica que el desempeño de cierta calidad frente a una situación clínica no permite predecir la calidad del desempeño frente a otra situación” (p. 7). El que el alumnado sea competente en la resolución de un caso no significa que sea competente en la resolución de todos los casos clínicos que se le presenten. Por esto, Brailosvsky, (2001) sugiere, en relación 
con la evaluación en la competencia clínica médica, disponer de muestreos numerosos que involucren suficientes contenidos y dimensiones. Aclara, sin embargo, que al aumentar el muestreo, no es conveniente la superposición de contenidos a través de los diferentes problemas clínicos, más bien estos pueden tener partes independientes y otras en común. Si se sigue este criterio, se puede afirmar que, para alcanzar la confiabilidad en las pruebas realizadas en el área clínica, es recomendable aumentar la varianza, por ejemplo, con el aumento de casos tratados y observados, no solo en número sino también en variedad.

En caso de realizar exámenes escritos, orales o de ejecución, la elaboración debe ser cuidadosa; esto se refiere, entre otros, a que sea acorde con lo temas abarcados en la enseñanza y, además, en la estructura y escogencia de los ítems. Si se realizan observaciones de las acciones llevadas a cabo en la clínica, se deberá recurrir a otro tipo de instrumentos para recogida de datos, como la lista de cotejo, las escalas de calificación y las rúbricas, en caso que además se desee ofrecer una retroalimentación escrita que pueda favorecer el aprendizaje del estudiantado.

La validez, también es otro elemento importante de ser tomado en cuenta. Se dice que un instrumento de evaluación es válido, “... cuando evalúa lo que se pretende evaluar con él” (Camilloni, Celman y Litwin, 1998, p. 76), es decir, un instrumento de evaluación tendrá validez cuando brinde la evidencia suficiente para decidir si lo que mide, corresponde a aquello para lo que fue diseñado. La calidad de una forma de evaluación dependerá del grado de pertinencia con el objeto evaluado (Camilloni, Celman y Litwin, 1998). Es, entonces, importante que las pruebas se relacionen con los objetivos planteados al inicio de las experiencias clínicas de aprendizaje, tanto en su contenido como en su forma. Para la evaluación de la competencia clínica, es importante que ....el instrumento utilizado debe de ser congruente con la estrategia metodológica utilizada y la habilidad que se desea medir" (Brailosky, 2001, p. 8).

El objeto de evaluación varía en la clínica, en este caso, no es simplemente conocer ni solamente el de aplicar los procedimientos, es necesaria la integración de estos dos, ejemplificados en el desempeño del estudiantado. La forma de evaluar debe ser acorde con los objetivos propuestos; si estos son procedimentales, sería recomendable hacer observaciones; más si estos se refieren al conocimiento de protocolos, teorías u datos de laboratorio, serían convenientes pruebas escritas u orales. Es importante darle la debida atención a este aspecto, porque la incongruencia entre lo enseñado y lo evaluado, como afirma Brailosky (2001), no favorece el impacto que podría tener la educación recibida.

Brown y Glasner, (2003) recomiendan que para evaluar la competencia del estudiantado, en la práctica profesional, se requiere de un tipo de evaluación no tradicional, ya que carece de sentido utilizar una evaluación tradicional de la práctica, pues puede que se desatiendan otros elementos importantes. Para evaluar habilidades, según las autoras, es necesario recurrir a diferentes fuentes de información, entre otras, a la observación, el uso de proyectos, el estudio de casos, los cuadernos de notas, los diarios reflexivos, el portafolio; todos importantes como métodos evaluativos.

Usar variedad de métodos en vez de solo exámenes escritos tradicionales puede proporcionar "...mayor confianza en las capacidades practicas de nuestros estudiantes." 
(Brown y Glasner, 2003, p.118). En aquellos casos en que la mayor parte de experiencias de aprendizaje clínicas sean prácticas, los métodos evaluativos deben ser acordes con ellas.

Es importante que, en los diferentes escenarios, el personal docente esté consciente de la importancia de la congruencia entre los objetivos, la forma de enseñar y lo evaluado, además de lo que entiende por evaluación, de cuándo aplicarla y lo que se pretende lograr con esta. La obtención de la información que se desea evaluar es parte del proceso educativo, recayendo su riqueza y dificultad más grande en interpretar, reflexionar y emitir juicios de los resultados y trabajar con estos (Camilloni, Celman y Litwin, 1998).

\section{¿Q̣ué evaluar en la clínica?}

Después de lo expuesto en el párrafo anterior, se debe plantear la interrogante: ¿Qué se debe evaluar en la clínica? Es un poco aventurado considerar que es posible realizar un listado de aprendizajes a alcanzar en la clínica afín a todas las carreras del área de la salud. Sin embargo, se pueden plantear ciertas propuestas desde algunos puntos comunes, los cuales, aunados al análisis propio de cada disciplina, pueden ofrecer acercamientos pertinentes para el desarrollo del área. El análisis lo deben hacer las expertas y los expertos de la disciplina en cuestión, de acuerdo con las intenciones educativas planteadas para cada caso. Los requerimientos en el desarrollo de las habilidades dependerán mucho del marco curricular de cada institución, del programa, del contexto y de la revisión del perfil profesional; no obstante, para este ensayo se presentan algunos planteamientos contenidos en documentos recuperados de la red. Uno de ellos es la propuesta de Greiner y Knebel (2009), quienes plantean cinco competencias para las profesionales y los profesionales del área de salud: brindar una atención centrada en la atención a la persona paciente, trabajar en grupos de trabajo interprofesionales, emplear una práctica basada en la evidencia, aplicar la mejoría en la calidad y, por último, utilizar la informática.

Además, la Organización Mundial de la Salud propone, para profesionales de la salud del siglo XXI, la competencia de la atención centrada en la persona paciente, la mejora de la calidad de la atención y el uso de las tecnologías entre otras (World Health Organization (WHO), 2005). Estas propuestas plantean el desarrollo del razonamiento clínico, la ejecución eficiente de los procesos clínicos, la comunicación efectiva con pacientes y sus familiares, el intercambio con pares y el profesionalismo como habilidades que mejoran la calidad en la atención, y que resultan importantes dentro del aprendizaje en el área. A continuación, se presentan apartados sobre cada aspecto en particular:

a. La comunicación con cada paciente es necesaria. Esta debe ser aprendida, practicada y evaluada. En esta habilidad se pueden incluir diferentes puntos como la forma de realizar una historia clínica, relación que se establece entre estudiante y paciente, el cómo se le comunican las noticias importantes a la persona paciente o cómo se indagan aspectos claves con la familia, la toma de decisiones, la promoción de la salud y los estilos de vida saludables, el cuidado farmacológico, los cuidados en etapas terminales para controlar el dolor, entre otros (Greiner y Knebel, 2009).

$\mathrm{Al}$ comunicarse, además, las futuras personas profesionales en ciencias de la salud requieren aprender a poner en palabras sencillas los conocimientos científico- médicos, 
para que sus pacientes les entiendan. También deben comunicar, de una forma asertiva, la necesidad de tomar decisiones al proponer tratamientos, en los que, con carácter obligatorio, se requiere una intervención profesional.

Es importante, en este punto, la mejoría en la comunicación entre las personas integrantes de equipos de trabajo interdisciplinarios, esta permitirá al alumnado practicar las habilidades necesarias para su futura labor profesional. Por ejemplo, el cuerpo médico requerirá tener una buena comunicación con el personal de un hospital o clínica, asimismo el equipo de enfermería entre sí y con el resto de profesionales de la salud en general, práctica que podría extenderse con otras personas de otras áreas como fisioterapia, nutrición, farmacia, microbiología, entre otros. También, para el caso de las futuras odontólogas y los futuros odontólogos, será importante aprender a comunicarse con otras personas no solo dentro del ámbito de su disciplina, sino también en abordajes integrales para la atención a pacientes.

La interrelación que se dé entre el estudiantado podría propiciar una experiencia enriquecedora para alcanzar esta habilidad comunicativa. Estas personas pueden cuestionarse: ¿Cuán efectiva es esta comunicación? ¿Qué acciones se deben realizar para mejorar el desarrollo de canales de comunicación entre las personas que ejercen roles en el proceso de atención? Las respuestas a estas cuestiones deben ser evacuadas por medio de los resultados obtenidos en evaluaciones conducentes a la mejoría continua del desempeño estudiantil.

La comunicación debe relacionarse, asimismo, con el desarrollo del razonamiento clínico, ya que, de esta forma, el estudiantado aprenderá a asociar la importancia de los aspectos biomédicos y los psicosociales en el cuidado del paciente (Windish, Price, Clever, Magaziner y Thomas, 2005). Además, para que el alumnado mejore el razonamiento clínico es necesario que contraste la evidencia científica y la evidencia clínica. En la interacción con otras y otros estudiantes de la salud, se enriquece también el razonamiento clínico, con un estudiantado más proactivo, capaz de generar ideas con base en las experiencias, con el uso del pensamiento crítico y a través de la evaluación de su propio desempeño y de las demás personas e inclusive de investigaciones en el campo.

b. El razonamiento clínico es otra habilidad para aprender y para evaluar y esta se refiere a una forma de pensar, y se relaciona con la toma de decisiones en la práctica profesional para orientar las acciones prácticas (Higgs, Jones, Loftus, Christensen, 2008); entre las habilidades en las que se deben entrenar los grupos en formación de profesionales de la salud, está la reflexión sobre su propia práctica, lo que puede permitirles motivarse para mantener y profundizar sus competencias profesionales (Durante, 2012) y la reflexión sobre las experiencias de otras y otros.

El razonamiento clínico es considerado como una habilidad fundamental en la medicina, incluso se le considera su médula espinal (Villaroel, Ribeiro y Bernal, 2014). Este proceso mental permite a las personas del área de medicina emitir un diagnóstico y brindar un pronóstico (Gormaz y Brailovsky, 2012). Sin embargo, este razonamiento no es exclusivo para la medicina, ya que se requiere también en las otras profesiones del área de la salud, en las que el establecimiento adecuado de las relaciones entre los signos, síntomas y el análisis permite ofrecer un diagnóstico certero, un tratamiento pertinente y un pronóstico fiable, con 
el desarrollo del criterio clínico y no la simple interpretación de lo observado. Esta habilidad es, además, contenido dependiente (Gormaz y Brailosvky, 2012), idea que el autor de este ensayo secunda y relaciona con el conocimiento de cada una de las diferentes disciplinas, sin embargo, la orientación que se dé para la evaluación de esta dependerá de las acciones que cada profesional realice.

Ser capaz de identificar los propios retos por parte del estudiantado, para analizarlos o discutirlos con sus pares o con su docente permitirá aclarar aquellos aspectos que generen dudas y alcanzar el enlace entre lo conocido y lo que queda por conocer para su posterior aplicación en la práctica, según el concepto de la zona de desarrollo próximo de Vigotsky, (1988), es decir, acercando el nivel de resolución de problemas del estudiantado al potencial con la supervisión de esa persona que posee mayor experiencia en el área: el personal docente. Como mencionan Gormaz y Brailosvky, (2012), "Es necesario que el estudiantado comprenda por qué cometió el error y dar una retroalimentación cognitiva adecuada para que transfiera lo aprendido a su práctica" (p. 17).

Se puede aprender de los errores, el comprender por qué se debe mejorar una u otra acción permite que el estudiantado autoevalúe su proceder y así mejore su práctica.

La práctica, el aprendizaje y la evaluación del razonamiento clínico son necesarios para el desarrollo de la experticia que permitirá a las futuras personas profesionales desenvolverse, en todo momento, en su disciplina, pues identifican aquellas acciones que pueden mejorarse en la ejecución de los procesos y así obtener resultados óptimos.

c. Ejecución de los procedimientos que se desarrollan en las prácticas clínicas de cada una de las profesiones de la salud. El estudiantado requiere, además de hacer las prácticas, de la retroalimentación pertinente por parte del profesorado tan pronto como sea posible después de ejecutar la acción. La evaluación de estos procesos es recomendable que sea formativa, es decir, que genere la retroalimentación que conduzca a mejorar el desempeño y a así potenciar el aprendizaje de cada estudiante, y no solo que se aplique con sentido sumativo de certificación. Se puede pensar en una alumna o un alumno que ha estudiado el proceso muchas veces en teoría e inclusive lo ha practicado en simuladores de diferente nivel, sin embargo, en el espacio clínico es la primera vez que ejecuta la acción en una situación real.

Se pueden plantear algunas interrogantes para la reflexión docente como: ¿Qué dificultades se han experimentado durante la práctica? ¿Cuáles acciones le son desconocidas al estudiantado? ¿Trata el estudiantado de resolver un problema práctico con conceptos y pasos memorizados antes de tener la experiencia? ¿Cuántas veces deberá realizar el proceso clínico antes de alcanzar la experticia? ¿Qué dificultades se presentan ahora que el estudiantado siente, ve, escucha y huele, durante su experiencia clínica? Las respuestas a estas preguntas pueden propiciar, en la docente y el docente, el sustento para la reflexión que permita delimitar los puntos a evaluar e identificar aquellos otros aspectos que deben estudiarse para dirigir al estudiantado hacia la mejora continua. Algunas de estas prácticas son propias de cada disciplina y deben ser analizadas y estudiadas por profesionales de cada área docente, por ejemplo, en enfermería la toma de vías o la administración y preparación de un fármaco, o en odontología la toma de una impresión de coronas y puentes, asimismo en 
medicina la sutura de una herida, el realizar un examen físico y, así, según cada carrera, con los procedimientos relacionados en cada una de las áreas de la salud.

Las prácticas profesionales son necesarias y también lo es la evaluación de estas; si no se evalúan adecuadamente, se pueden hacer las cosas mal una y otra vez, sobre todo si no se recibe la retroalimentación pertinente en el momento preciso, lo que puede marcar la diferencia en la mejora del ejercicio. Será necesario que el cuerpo docente reflexione sobre los aspectos a evaluar en cada práctica, además de la forma de evaluación, recordando que debe existir congruencia entre los objetivos propuestos en el curso y la forma de evaluar, asimismo al nivel en el que el estudiantado se encuentre ubicado -sea este, principiante, intermedio $u$ avanzado- en sus respectivas carreras.

d. Profesionalismo es el componente actitudinal al que se le atribuyen acepciones diferentes relacionadas como “... las actitudes y la disposición manifestado [sic] de algún modo...” (Letelier y Velandia, 2010, p. 2), o también, “... conjunto de principios éticos y deontológicos, valores y conductas que sustentan el compromiso de los profesionales de la medicina con el servicio a los ciudadanos..." (Organización Médica Colegial de España, 2010, p. 16). Esta última definición se acuño para profesionales del área de la medicina, sin embargo, es aplicable a las otras carreras en las cuales se brinda atención a pacientes de una u otra forma e, inclusive, a las demás profesiones, no necesariamente relacionadas con el área de la salud.

Es necesario que en cada disciplina se reflexione sobre cuáles son los atributos que se van a evaluar y qué uso se les dará a los resultados. Es importante que los procesos por medio de los cuales se evaluará el comportamiento profesional se basen en el propósito que se le dará a la evaluación (Hawkins, Katsufraquis, Holman y Clauser, 2009). Se deben evaluar los objetivos actitudinales propuestos y corresponder con acciones metodológicas utilizadas por los grupos profesionales en el proceso de enseñanza y aprendizaje.

La observación es una de las herramientas que permite evaluar este aspecto en las futuras personas profesionales del área de la salud. Los contenidos procedimentales y los cognitivos son diferentes a los actitudinales, abarcados en el profesionalismo, por lo que los instrumentos utilizados para evaluar el conocimiento, y las habilidades clínicas no pueden ser iguales a los empleados para la evaluación del profesionalismo (Hawkins, Katsufraquis, Holman, y Clauser, 2009). Otros aspectos importantes a tomar en cuenta para mejorar la confiabilidad en la evaluación son, por ejemplo, el que sea ejecutada por varios evaluadores, en variados casos, aunado a que sean suficientes los aspectos a tomar en cuenta, en este último punto que no sean tantos que desanimen a la persona evaluadora a llevar a cabo la observación, en este caso no más de diez (Norcini, 2003) y que exista la debida correspondencia como ya se mencionó, entre los objetivos y las metodologías utilizadas de enseñanza.

\section{La retroalimentación y su importancia}

La evaluación puede tener diferentes funciones como ya se ha mencionado. Estas, determinadas por distintos factores como son, por ejemplo, el momento en que se realice, también de acuerdo con el propósito de quien o quienes la diseñan, además de los criterios con 
que se estructura (objetivos, metodologías. Los resultados obtenidos a través de esta constituyen más allá de una serie de datos inconexos, una fuente de información útil, para la toma de decisiones, tanto para el grupo docente como para el estudiantado, además de la promoción en el curso. La retroalimentación corresponde a la acción de brindar información al estudiantado sobre los resultados de la evaluación, de manera que esta o este sean capaces de buscar la solución o junto con sus docentes encontrar la ruta para solventar la falencia hallada. Es así como, en la docencia, estos datos pueden utilizarse para tomar decisiones con respecto a los cambios necesarios a realizar o de conductas que se deban reforzar en la enseñanza, en aras de obtener una mayor calidad en el aprendizaje y así en el desempeño estudiantil.

La retroalimentación, para el estudiantado, es fundamental para la orientación que le permita la superación y, de esta manera, lograr un proceso que le permita desarrollarse de forma continua en su área. En la retroalimentación, sea cual sea el tipo de evaluación, es mejor si se siguen ciertos principios (Davis, Forrest, y Mc Kimm, 2013), como lo son:

a. Auténtica, con un propósito definido para promover la mejoría, y lejano para minar la relación del estudiantado y el profesorado.

b. A tiempo, es decir, tan pronto como sea posible después del evento. En el caso de un examen de selección, la retroalimentación puede tardarse algunos días, sin embargo, en la clínica la retroalimentación efectiva debe ofrecerse tan pronto como sea posible (Cantillon y Sargeant, 2008; Davis, Forrest y Mc Kimm, 2013).

c. Referida a comportamientos no a aspectos personales. Además, relacionada con comportamientos específicos y no al desempeño en general, como por ejemplo: Un "Bien hecho", sin referirse a un hecho en particular, es recomendable que abarque lo más uno o dos aspectos a la vez (Cantillon y Sargeant, 2008).

d. Brindarla en presente, no referida a falencias pasadas. Es decir, no recalcar conductas que han sido mejoradas o de las cuales el estudiantado viene realizando esfuerzos por superar.

e. Ser personal o individual. No realizar generalizaciones que pueden no ser comprendidas por las personas que lo requieren.

f. Debe haber congruencia entre el mensaje y los gestos de expresión que se hagan al realizar la corrección. El equipo docente debe asegurarse de que el estudiantado comprenda lo que se le explica. Además, el lenguaje no debe poseer elementos de juicio (Cantillon y Sargeant, 2008).

El recibir muy poca retroalimentación o no recibirla del todo es poco útil para el estudiantado (Davis, Forrest y Mc Kimm, 2013). Se han sugerido algunas técnicas para brindar la retroalimentación, como por ejemplo, la del sandwich donde se menciona primero un aspecto positivo en el desempeño del estudiantado, luego uno negativo y finalmente uno positivo (Cantillon y Sargeant, 2008). Si bien propuestas como esta no deben tomarse como una receta de cocina, cada docente deben tener claro que el arte de brindar la retroalimentación es crítica en la docencia y que esta cualidad se adquiere con la práctica (Cantillon y Sargeant, 2008). 
La retroalimentación en la educación superior es primordial en el desarrollo de un aprendizaje efectivo, y con estudiantes del área de la salud que participan en los escenarios clínicos es necesaria, para así reforzar un buen desempeño y evitar las pobres prácticas a expensas de pacientes y colegas (Cantillon y Sargeant, 2008).

\section{Conclusiones}

El proceso enseñanza aprendizaje en los escenarios clínicos difiere de otros no solo por las características propias de las disciplinas del área de la salud, sino también por su dinámica, que involucra la variabilidad, la impredictibilidad y la inmediatez en la toma de decisiones. No siempre es posible establecer las experiencias de aprendizaje que pueden enmarcarse en un planeamiento de intenciones educativas. Se presentan, además, en estos espacios, tensiones propias de la atención de pacientes, ya que participan tres personas como actrices/ actores a la vez, estudiante, docente y paciente. Cada cual tendrá sus necesidades y expectativas: para el estudiantado no solo aprender sino también pasar el curso; las docentes y los docentes cumplir con el desarrollo de un curso y enseñar, además, brindar atención a cada paciente que se trata, dentro de un marco de atención profesional y ético que asegure el respeto y la integridad física para la persona que se está asistiendo. Y como paciente, se busca la atención, por una dolencia cuya resolución requiere un cuidado inmediato, que debe ser de calidad y efectivo; además, estas personas pueden tener otros intereses que consideran importantes como seres biopsicosociales que son.

La evaluación en los espacios clínicos de enseñanza requiere ser repensada, ya que no solo permite la promoción del alumnado, sino además debe brindar la retroalimentación sobre el desempeño discente que le guíe al mejoramiento continuo de sus acciones.

En la clínica, la pregunta sobre ¿qué evaluar?, no solo corresponde al nivel cognitivo, no bastan solo los exámenes tradicionales, sino que es necesaria la integración de la teoría y la práctica aplicada a la atención de pacientes, relacionadas con otras habilidades como son: la capacidad de comunicación con pacientes y entre profesionales de otras disciplinas de salud, el razonamiento clínico, lo que permitirá el desarrollo de la experticia, el reforzamiento en la toma de decisiones, resolución de problemas y el contraste entre la evidencia clínica y científica, asimismo, la ejecución de procedimientos y el profesionalismo. Por todo esto se requiere reformular los métodos evaluativos congruentes con lo que se va a evaluar.

Y sobre el interrogante que se plantea en el título sobre ¿por qué evaluar?, la evaluación requiere de la atención del personal docente en la clínica no solo para certificar y codyuvar en su crecimiento formativo, el cual es muy importante para que el estudiantado desarrolle la metacognición, mejore la autocrítica y la superación, entre otros.

También es importante el cómo brindarla, es decir, el momento y la forma de ejecutarla, ya que esto permitirá que sea fructífera en el aprendizaje del alumnado, aspectos como la confiabilidad, la validez, la objetividad y la representatividad deben tomarse en cuenta y motivar, con su aplicación, al perfeccionamiento del desempeño y el mejoramiento de calidad profesional dentro de un marco de respeto por todos los seres humanos, tanto pacientes, como colegas y profesionales de áreas similares. 
Las ideas discutidas en este ensayo permitirán la reflexión y el análisis de las personas profesionales docentes del área, con el propósito de mejorar su desempeño en la pedagogía universitaria y la calidad de las graduadas y los graduados en cuyas manos estarán, a la larga, nuestras propias vidas.

\section{Referencias}

Álvarez, C., Rojas, T., Navas, R. y Quero, M. (2011). Evaluación del aprendizaje en contextos clínico- odontológicos. Ciencia odontológica, 8, 112-119. Recuperado de www. actaodontologica.com/ediciones/2007/3/pdf/ambientes aprendizaje.pdf

Álvarez, L., Gulgelmeier, V. y Hermida, L. (2013). Cómo aprenden los estudiantes de odontología que cursan el ultimo año de la carrera? Odontoestomatología, 15 (21). Recuperado de http://www.scielo.edu.uy/scielo.php?script=sci arttext\&pid=S1688-93392013000100002

Boshuiizen, H., Norman, G. y Schmidt, H. (1990). A cognitive perspective on medical expertise: theory and implication [Una perspectiva cognitiva sobre la experiencia médica: teoría e implicación] Journal of the Association of American Medical Colleges, 65 (10). Recuperado de http://journals.lww.com/academicmedicine/Abstract/1990/10000/A cognitive perspective on medical expertise .1.aspx

Borrel, R. M. (2005). La educación médica en América Latina: debates centrales sobre los paradigmas científicos y epistemológicos. En R. Chiara (Dir.). Proceso de transformación Curricular: otro paradigma es posible. Universidad Nacional de Rosario. Argentina: Editorial de la Universidad Nacional de Rosario. Recuperado de https://cursos. campusvirtualsp.org/pluginfile.php/86391/mod resource/content/0/Modulo I/Capitulo EM para libro de Rosario.pdf

Brailosky, C. (2001). Educación médica, evaluación de las competencias. Recuperado de http://www.esm.ipn.mx/Documents/rediseno curricular/paginas/DocumentosDeApoyo/ EducacionMedicaEvalCompetencias.pdf

Brown, S. y Glasner, A. (2003). Evaluar en la universidad. Problemas y nuevos enfoques. España: Editorial Narcea.

Bruera, R. (2008). Manual de didáctica de la medicina. Argentina: Buena Vista Editores.

Camilloni, A., Celman, S. y Litwin, E. (1998). La evaluación de los aprendizajes en el debate didáctico contemporáneo. Ecuador: Editorial Paidós. 
Cantillon, P. y Sargeant, J. (2008). Giving feedback in clinical settings. British Medical Journal, 337 (7681). Recuperado de www.bmj.com/content/337/bmj.a1961

Davis, M., Forrest, K. y McKimm, J. (2013). How to Assess doctors and Health [Cómo evaluar a médicos y profesionales de la salud] Professionals. Reino Unido: Editorial John Wiley and Sons. doi: https://doi.org/10.1002/9781118523469

Delany, C. y Molloy, E. (2009). Clinical Education in Health Professions [Educación Clínica en Profesiones de la Salud.]. Australia: Elsevier.

Delgado, K. (2009). Evaluación y metacognición en el aula. Revista Investigación Educativa, 13 (24) Recuperado de http://sisbib.unmsm.edu.pe/bibvirtual/publicaciones/inv educativa/2009 n24/pdf/a06v13n24.pdf

Diccionario de la Real Academia Española (2015). Clínica. Recuperado de http://dle.rae.es

Diccionario Etimológico. (2015) Clínica. Recuperado de http://etimologias.dechile.net/?cli.nica

Díaz-Barriga, F. y Hernández, G. (2007). Estrategias docentes para un aprendizaje significativo. Una interpretación constructivista. México, D.F.: Editorial Mc Graw Hill.

Durante E. (2012). La enseñanza en el ambiente clínico: Principios y métodos. Revista de Docencia Universitaria, 10 (Número especial), 149-175. Recuperado de http://red-u.net/ redu/files/journals/1/articles/468/public/468-1481-2-PB.pdf

Escalante, R. y Matos, G. (2013). Simulación Clínica: Seguridad y Calidad para el paciente. Interciencia, 4 (1), 41-48 Recuperado de http://www.clinicainternacional.com.pe/pdf/ revista-interciencia/9/articulo_revision.pdf

Flores, F., Contreras, N. y Martínez, A. (2012). Evaluación del aprendizaje en la educación médica. Revista de la facultad de Medicina de la UNAM, 55 (3), 42-48. Recuperado de http://www.medigraphic.com/pdfs/facmed/un-2012/un123h.pdf

Greiner, A. y Knebel, E. (2009). Health Professions Education: A Bridge to Quality [Educación de las profesiones de la salud: un puente hacia la calidad]. Institute of Medicine of the National Academies. Washington D.C.: Autor.

Gormaz, C. y Brailosvky, C. (2012). Desarrollo del razonamiento clínico en medicina. Revista de docencia universitaria, 10 (Número especial), 177-199. Recuperado de http://red-u.net/ redu/index.php/REDU/article/view/469 
Hawkins, R. E., Katsufraquis, P. J., Holman, M. C. y Clauser, B. E. (2009). Assessment of medical professionalism: Who, What, When, Where, how, and...why? [Evaluación de la profesionalidad médica: ¿Quién, qué, cuándo, dónde, cómo y ... por qué?] Medical Teacher, 31, 385-398. Recuperado de http://www.ncbi.nlm.nih.gov/pubmed/19404894

Higgs, J., Jones, M., Loftus, S. y Christensen, N. (2008). Clinical reasoning in the Health Professions Razonamiento clínico en las profesiones de la salud]. Inglaterra: Elsevier.

Letelier, V. y Velandia, A. (2010). Profesionalismo en enfermería, el hábito de la excelencia del cuidado. Avances de Enfermería, 28 (2), 145-158. Recuperado de www.scielo.org.co/scielo. php?pid=S012145002010000200013\&script=sci abstract\&tlng=es

Liljedhal, M., Engqvist, L., Porthén, Ch. y Bolander, K. (2014). What students really learn: contrasting medical and nursing students'experiences of the clinical learning environment [Lo que realmente aprenden los estudiantes: contrastar las experiencias de los estudiantes de medicina y enfermería en el entorno de aprendizaje clínico]. Advance in health Sciencies Education. doi: https://dx.doi.org/10.1007/s10459-014-9564-y

López, B. y Hinojosa, E. (2001). Evaluación del aprendizaje. México: Trillas.

Lucarelli, E., Finkelstein, C., Donato, M., Calvo, G., Del Regno, P., Gardey, M., Nepomneschi, M. y Solberg, V. (2009). La enseñanza de prácticas profesionales en la universidad: Estilos docentes diversos para aprendizajes complejos en las clínicas odontológicas. Profesorado, Revista de currículum y formación del profesorado, 13 (1), 1-18. Recuperado de www.ugr.es/ recfpro/rev131COL1.pdf

Man, K., Gordon, J. y MacLeod, A. (2004). Reflection and reflective practice in health professions education a sistematic review. Avd in Health Sciences Education, 14, 595621. doi: https://doi.org/10.1007/s10459-007-9090-2

Moreno, T B. (2009). La evaluación del aprendizaje en la universidad. Tensiones, contradicciones y desafíos. Revista Mexicana de Investigación Educativa, 14 (41), 563-591. Recuperado de http://www.redalyc.org/pdf/140/14004110.pdf

Norcini, J. (2003). Peer assessment of competence [Evaluación por pares de la competencia]. Medical Education, 37, 539-543. doi: https://doi.org/10.1046/j.1365-2923.2003.01536.x

Organización Médica Colegial de España. (2010). Profesión médica, profesional médico, profesionalismo médico. Recuperado de http://cmpont.es/pdfs/OMCprofesionmedica.pdf 
Quirk, M. (2006). Intuition and Metacognition in Medical Education: Keys to developing expertise [Intuición y metacognición en la educación médica: claves para el desarrollo de la experiencia]. Estados Unidos: Springer Publishing Company.

Santamaría, M. (2005). ¿Cómo evaluar los aprendizajes en el aula? San José, Costa Rica: Editorial de la UNED.

Stailmeijer, R., Dolmans, D., Wolfhagen, I. y Scherpbier, A. (2009). Cognitive Apprendiceship in clinical practice: can it stimulate learning in the opinion of students? [Aprendizaje cognitivo en la práctica clínica: ¿puede estimular el aprendizaje en la opinión de los estudiantes?], Adv in Health Sci Educ, 14, 535-546. Recuperado de www.ncbi.nlm.nih. gov/pmc/articles/PMC2744784/

Sweet, J., Huntly, S. y Taylor, I. (2003). Effective learning and teaching in Medical, Dental and Veterinary Education [Aprendizaje y enseñanza efectivos en la Educación Médica, Dentaly Veterinaria]. England: Kogan Page Limited. doi: https://doi.org/10.4324/9780203416860

Taylor, C., Grey, N. y Satterwaite, J. (2013). Assessing the Clinical of Dental Students: A Review of the Literature [Evaluación de la clínica de los estudiantes de odontología: una revisión de la literatura]. Journal of Education and Learning, 2 (1), 20-31. doi: https:// doi.org/10.5539/jel.v2n1p20

Vigotsky, L. (1988). El desarrollo de los procesos psicológicos superiores. España: Grijalbo.

Villaroel, J., Ribeiro, Q. y Bernal, N. (2014). Razonamiento clínico: Su déficit actual y la importancia del aprendizaje de un método durante la formación de la competencia clínica del futuro médico. Rev Cient Cienc Méd, 17 (1). 29-36. Recuperado de http://www. redalyc.org/pdf/4260/426041228009.pdf

Windish, D., Price, E., Clever, S., Magaziner, J. y Thomas, P. (2005). Teaching Medical Students the important connection between communication and clinical reasoning [Enseñanza de los estudiantes de medicina la conexión importante entre la comunicación y el razonamiento clínico]. Journal of General Internal Medicine, 20 (12), 1108-1113. doi: https://doi.org/10.1111/j.1525-1497.2005.0244.x

World Health Organization (WHO). (2005). Preparing a health care workforce for 21st century [Preparación de una fuerza de trabajo de salud para el siglo XXI]. World Health Organization. Francia. Recuperado de http://www.who.int/chp/knowledge/publications/ workforce report.pdf 